\title{
A Linear Model Method for Biodiversity-Ecosystem Functioning Experiments
}

\author{
Thomas Bell, ${ }^{1 *}$ Andrew K. Lilley, ${ }^{2}$ Andy Hector, ${ }^{3}$ Bernhard Schmid, ${ }^{3}$ Lindsay King, ${ }^{4}$ and \\ Jonathan A. Newman ${ }^{4}$
}

1. Department of Zoology, University of Oxford, South Parks Road, Oxford OX1 3PS, United Kingdom; 2. Pharmaceutical Science Research Division, King's College London, 150 Stamford Street, London SE1 9NH, United Kingdom; 3. Institute of Environmental Sciences, University of Zurich, Winterthurerstrasse 190, CH-8057 Zurich, Switzerland; 4. School of Environmental Sciences, University of Guelph, Guelph, Ontario N1G 2W1, Canada

Submitted March 23, 2009; Accepted August 5, 2009; Electronically published October 20, 2009

Online enhancements: appendixes.

\begin{abstract}
Aвstract: Experiments that manipulate species richness and measure ecosystem functioning attempt to separate the effects of species richness (the number of species) from those of species identity. We introduce an experimental design that ensures that each species is selected the same number of times at each level of species richness. In combination with a linear model analysis, this approach is able to unambiguously partition the variance due to different species identities and the variance due to nonlinear species richness, a proxy measure for interactions among species. Our design and analysis provide several advantages over methods that are currently used. First, the linear model method has the potential to directly estimate the role of various ecological mechanisms (e.g., competition, facilitation) rather than the consequences of those mechanisms (e.g., the "complementarity effect"). Second, unlike other methods that are currently used, this one is able to estimate the impact of diversity when the contribution of individual species in a mixture is unknown.
\end{abstract}

Keywords: random partitions design, biodiversity, ecosystem functioning, linear model.

\section{Introduction}

Among the principal goals of community and ecosystem ecology is to understand how species contribute to ecosystem processes such as the rate and stability of nutrient cycling (Hooper et al. 2005; Balvanera et al. 2006). The issue is also of conservation interest because species loss might have adverse impacts on the remaining community or on human society (Hector et al. 2001; Srivastava and Vellend 2005). As a consequence, experiments that manipulate species richness (the number of species) and measure ecosystem functioning (e.g., productivity) have be-

* Corresponding author; e-mail: thomas.bell@zoo.ox.ac.uk.

Am. Nat. 2009. Vol. 174, pp. 836-849. (C) 2009 by The University of Chicago. 0003-0147/2009/17406-51165\$15.00. All rights reserved.

DOI: $10.1086 / 647931$ come common over the past decade (Balvanera et al. 2006; Cardinale et al. 2006).

The original experiments in the field generated some controversy surrounding the design and statistical analysis of the experiments and the degree to which mechanisms can be inferred from the results (Aarssen 1997; Huston 1997; Tilman et al. 1997; Wardle 1998, 1999; Huston et al. 2000). When the species richness of a community is manipulated, it is not independent of the manipulation of the presence or absence of particular species in the community (Schmid et al. 2002a). As a consequence of this constraint on the way in which biodiversity experiments can be designed, analyses of the experiments are hampered by the difficulty of separating the impact of species richness on ecosystem functioning from the impact of constituent species. If the presence or absence of each species in an experimental community is considered as a separate experimental factor, then a full factorial experiment design contains all possible species compositions that can be derived from a species pool. This design is the preferred design because it gives the maximum amount of information about how composition and richness affect ecosystem functioning. However, such experiments quickly become intractable because the number of compositions accelerates with increasing species richness. The full factorial design might be reasonable for the simplest experiments that use just a few species, but it is clearly impractical even for the most species-poor natural communities, which might contain dozens to hundreds of species. Useful experimental designs reflect this compromise between tractability and complete information by selecting a subset of the possible compositions.

The design that most recent large-scale experiments (Hector et al. 1999; Tilman 1999a) have used is to sample the $S$ available species randomly with replacement at each level of species richness. In the random draw design, for 
any particular mixture, there is a higher probability of selecting species with a large effect on ecosystem functioning, if one exists, at high compared with low levels of species richness (Aarssen 1997; Huston 1997; Huston and McBride 2002). If two of the species interact in some way, the probability that those species will be contained together in a composition (and therefore that the interaction occurs) increases as the number of species in the composition increases. The way in which the experiment is designed can therefore alter the conclusions, so the first purpose of this article is to investigate the consequence of using a different experiment design.

In addition to the issues surrounding the design of biodiversity experiments, there has also been much discussion of how biodiversity-ecosystem functioning experiments should be analyzed to infer ecological mechanisms (Loreau and Hector 2001; Schmid et al. 2002a; Fox 2005, 2006; Fox and Harpole 2008). For example, if species occupy distinct niches (e.g., feed on different resources), then species-rich communities will also be more productive because more of the total resource pool is utilized (the "complementarity" effect). Alternatively, species-rich communities might have higher levels of functioning because they are more likely to include competitively dominant species that also have relatively large effects on functioning (the "selection" effect; Tilman 1999b). The earliest experiments were ambiguous because they could not distinguish among ecological mechanisms, both because of deficiencies in the way the experiments were designed and because of a lack of analytical tools.

The initial biodiversity-ecosystem functioning experiments used the ANOVA framework (Hector et al. 1999). If there are $M$ unique species compositions in an experiment and the species richness of each composition is $R$, then the observed variation in ecosystem functioning can be partitioned into variation due to richness, variation due to compositions nested in richness, and residual variation (Schmid et al. 2002a). However, in most experimental designs_-which lack features of the design presented herethere are some notable drawbacks with this method of analysis. First, the analysis assumes that compositions are independent and so does not take into account that some pairs of species compositions share species or share more species than others (the "variance-reduction effect"; Huston 1997). Second, there is no insight into the relative importance of each species in determining the level of ecosystem functioning. The primary goal of the current study is to overcome these drawbacks.

Current methods have developed additional analyses outside of the ANOVA framework. Loreau and Hector (2001) introduced a method that partitions the net effect of species richness on ecosystem functioning into two terms that are interpreted as the effect of niche complementarity and the effect of selection on ecosystem func- tioning. There are three main drawbacks with the method. First, there is continuing debate as to the degree to which the model describes complementarity and selection effects (Petchey 2003; Fox 2005). This problem is surmountable, and there are continued efforts to develop these analyses to improve the ecological interpretation of the results (Fox 2005). Second, in these analyses, functioning of mixtures is compared to monoculture functioning, and thus, the level of functioning associated with every monoculture is required. Analyses that relax this constraint have recently been developed (Fox 2006), although other design constraints are imposed. Third, in order to perform the calculations for any of the approaches currently employed, it is necessary to measure the contribution of every species in a community to the particular ecosystem function of interest (e.g., biomass production). Obtaining this information is often difficult or impossible either because of practical constraints or because these are "emergent" properties of the communities (Fox and Harpole 2008). For example, if there is a positive relationship between species richness and stability (e.g., of community biomass), stability is an emergent property of the community, and it is unclear how to measure the contribution of every species to stability in polyculture. This last constraint is a particular hindrance to biodiversity-ecosystem functioning research because the contribution of each species to functioning cannot be measured for most functions of ecological and economic importance.

We describe an experimental design and corresponding linear model method that overcome these three difficulties. For experiments using the new design, the method is able to assess the influence of both species richness and species identity on ecosystem functioning without requiring knowledge of the contribution of individual species to ecosystem functioning in mixture.

\section{The Random Partitions Design}

We have developed an experimental design that randomly partitions the species pool at each level of diversity. In this design, every species is drawn at random from a pool of $S$ species without replacement, such that each species is selected exactly once at each level of diversity. Each composition is replicated, as is the process of selecting species without replacement. The design bears similarities to those presented in several other publications (Hodgson et al. 2002; Roscher et al. 2004, 2005) and was used in two previous experiments (Bell et al. 2005; Salles et al., forthcoming). A summary of the terminology is provided in appendix A in the online edition of the American Naturalist.

For an experiment with a species pool of $S$ species, let $\delta=\left\{R_{1}, R_{2}, \ldots, R_{d}\right\}$ be the set of all whole number factors 
of $S$, where $R_{1} \ldots R_{d}$ is the number of species at each of the $d$ richness levels. The vector $\delta$ therefore defines the species richness levels used in the experiment. For example, if $S=10$, then $\delta=\{1,2,5,10\}$ and $d=4$. It should be emphasized that although only factors of $S$ can be used, it is not necessary to use all species richness levels that could be used in the experiment; so, for example, including the monocultures is not required. Evidently, simply removing levels of richness would decrease the ability to detect some of the effects of interest. Eliminating levels of richness but increasing replication elsewhere in the experiment would increase the power of detecting effects but would decrease the generality of the results.

For any given element of $\delta$ there are $S / R$ compositions each with $R$ species present, so for $S=10$ and $R=2$ there are five different compositions. The species that make up the compositions are chosen at random by sampling the $S$ species without replacement such that every species is sampled exactly once from the species pool; so, for $R=3$ and $S=6$, the six species would be partitioned into two three-species compositions (Wacker et al. 2008). The complete process of constructing a set of experimental units is carried out independently $Q$ times (i.e., there are $Q$ "partitioned species pools"). For any given $S$, the total number of experimental units following this procedure is $\tilde{f}(S)=n Q \sum_{R \in \delta}(S / R)$. We illustrate the design in figure 1, using a pool of $S=6$ species. If the random partitions design is used in combination with the linear model approach that we outline below, it is possible to separate the impact of "nonlinear species richness" from the effect of species identity. As we will show below, the "nonlinear species richness effect" is a good proxy measure of both positive and negative species interactions.

The current method is not limited to additive or substitutive designs (Harper 1977) and stipulates only that the abundances of species be initially equal. This gives each species an equal chance of dominating the functioning of the community, and final species abundances would be expected to adjust accordingly. This would be an acceptable assumption for most plant communities where the law of constant yield (Harper 1977) applies but would otherwise have to be ascertained on a case-by-case basis. Alternatively, for short-term experiments where abundances do not have the opportunity to adjust, it might be possible to scale the species identity effects (see below) by a coefficient indicating their initial abundance, but we do not address this possibility in this study.

\section{Analysis of the Random Partitions Design}

\section{Model Structure}

In a biodiversity-ecosystem functioning experiment conducted according to the random partitions design (see

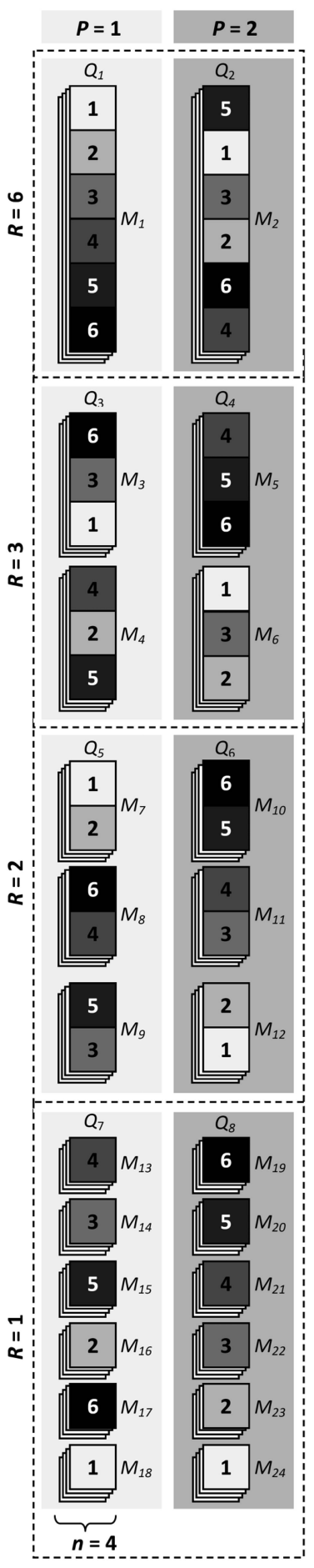

Figure 1: An illustration of the random partitions design. Each square is a species, with the numbers designating whether it is species $1,2,3$, 4,5 , or 6 . The variable definitions are given in appendix A in the online edition of the American Naturalist. 
above), for each of the $\tilde{f}(S)$ experimental units, each of the $S$ species is scored as being present or absent, and each of the $M$ compositions is independently replicated $n$ times. The least squares model can then be described by

$$
y=\beta_{0}+\beta_{R} x_{R}+\left(\sum_{i}^{S} \beta_{i} x_{i}\right)+\beta_{Q} x_{Q}+\beta_{M} x_{M}+e,
$$

where $y$ is the response variable (i.e., ecosystem functioning), $\beta_{0}$ is the intercept, $\beta_{R}$ is the effect of species richness on ecosystem functioning where richness is treated as a factor with $S$ levels (i.e., rather than as a continuous variable), $x_{R}$ codes for the particular level of species richness, $\beta_{i}$ is the effect of species $i$ on ecosystem functioning, $x_{i}$ codes whether species $i$ is absent $\left(x_{i}=0\right)$ or present $\left(x_{i}=1\right), \beta_{Q}$ is the effect of the particular "partitioned species pool," $x_{Q}$ codes for the partitioned species pool, $\beta_{M}$ is the effect of compositions, $x_{M}$ is a factor that codes for the particular composition, and $e$ is a vector of random errors. Each of the $i$ species coefficients can be gathered into a single term, $\sum_{i}^{S} \beta_{i} x_{i}$, which we interpret as the overall effect of species identities on ecosystem functioning. Each of the terms in equation (1) is associated with a corresponding sum of squares $\left(\mathrm{SS}_{0}, \mathrm{SS}_{R}, \mathrm{SS}_{1}, \mathrm{SS}_{2}, \ldots, \mathrm{SS}_{S}, \mathrm{SS}_{Q}\right.$, and $\mathrm{SS}_{M}$ ), which are calculated sequentially in the order in which they appear in the equation.

The principal difficulty with this method of analysis (which also exists for all approaches that use this formulation) is that the terms in equation (1) are not independent (i.e., the model is overdetermined). Consequently, the order in which the terms are entered into the model determines the outcome of the least squares fit. So, for example, if species richness is entered into the model before the species effects (as is shown in eq. [1]), then some portion of the sum of squares attributed to species richness $\left(\mathrm{SS}_{R}\right)$ cannot be attributed to species identity at the same time $\left(\mathrm{SS}_{I}\right)$. This dependence of the species identity term on the species richness term (or vice versa) is unavoidable because, for example, the species richness is known once the single species identities are known. Similarly, if we know the species richness and whether all but one of the species is present or absent, we immediately know whether the final species is present or absent. There is therefore an inevitable interdependence between $\mathrm{SS}_{R}$ and $\mathrm{SS}_{I}$ that causes their sums of squares-obtained by fitting each term first in separate models-to overlap. However, when the random partitions design is used, it is straightforward to quantify the degree to which $\mathrm{SS}_{I}$ and $\mathrm{SS}_{R}$ overlap. To do this, we break down the species richness term into linear and nonlinear contrasts,

$$
\begin{aligned}
\beta_{R} x_{R} & =\beta_{\mathrm{LR}} x_{\mathrm{LR}}+\beta_{\mathrm{NLR}} x_{\mathrm{NLR}}, \\
\mathrm{SS}_{\mathrm{R}} & =\mathrm{SS}_{\mathrm{LR}}+\mathrm{SS}_{\mathrm{NLR}},
\end{aligned}
$$

where $\beta_{\mathrm{LR}} x_{\mathrm{LR}}$ is the effect of linear richness (i.e., richness treated as a linear, untransformed, continuous variable), $\beta_{\mathrm{NLR}} x_{\mathrm{NLR}}$ is nonlinear species richness treated as a categorical variable with $S-2$ degrees of freedom and is the effect of richness not attributed to the linear richness term, and $\mathrm{SS}_{\mathrm{LR}}$ and $\mathrm{SS}_{\mathrm{NLR}}$ are the sums of squares associated with each of those terms. We can now rewrite equation (1) to reflect these new definitions:

$$
\begin{aligned}
y= & \beta_{0}+\beta_{\mathrm{LR}} x_{\mathrm{LR}}+\beta_{\mathrm{NLR}} x_{\mathrm{NLR}} \\
& +\left(\sum_{i}^{S} \beta_{i} x_{i}\right)+\beta_{\mathrm{Q}} x_{\mathrm{Q}}+\beta_{M} x_{M}+e .
\end{aligned}
$$

One feature of experiments that use the random partitions design is that the portion of $\mathrm{SS}_{R}$ that overlaps with $\mathrm{SS}_{I}$ is exactly equal to $\mathrm{SS}_{\mathrm{LR}}$. Consequently, once $\beta_{\mathrm{LR}} x_{\mathrm{LR}}$ has been entered into the model, the effect of species identity $\left(\sum_{i}^{S} \beta_{i} x_{i}\right)$ and the effect of nonlinear species richness $\left(\beta_{\mathrm{NLR}} x_{\mathrm{NLR}}\right)$ are orthogonal (i.e., do not share sums of squares). The formulation presented in equation (3) therefore provided two insights: first, it has explained the degree to which composition and richness overlap, and second, it has obtained species identity and richness terms that are orthogonal.

We have illustrated this process using a simple numerical example. First, we created a vector of ecosystem functioning values for each of the compositions shown in figure 1. For the purpose of this example, the ecosystem functioning values for each composition were calculated as

$$
y_{j}=\log _{10}\left(d_{j}\right)+e
$$

where $y_{j}$ is the functioning of composition $j, d_{j}$ is the species richness of composition $j$, and $e$ is the standard normal random error (mean $=0, \mathrm{SD}=1$ ). Without loss of generality, we assumed that none of the compositions were replicated (i.e., $n=1$ ). The data used in the following analysis, which are only one possible realization of the data, are plotted in figure A1 in the online edition of the American Naturalist. We first partitioned the total sum of squares into sums of squares due to compositions $\left(\mathrm{SS}_{M}\right)$, "partitioned species pools" $\left(\mathrm{SS}_{\mathrm{Q}}\right)$, and residual error (model A in fig. 2). The residual error is equal to 0 for this example because the $M$ compositions were not replicated.

When species richness and the individual species effects were added to the model in the order that they are presented in equation (1), $\mathrm{SS}_{R}$ explained part of the variance previously attributed to $\mathrm{SS}_{Q}$, and $\mathrm{SS}_{I}$ explained part of the 


\section{Demonstration that the degree to which $\mathrm{SS}_{I}$ and $\mathrm{SS}_{R}$ overlap is equal to $\mathrm{SS}_{\mathrm{LR}}$}

\begin{tabular}{|c|c|c|c|c|}
\hline Source of variance & df & F-den. & Model A Model B & Model C \\
\hline \multicolumn{5}{|l|}{ Species richness $\left(\mathrm{SS}_{R}\right)$} \\
\hline Linear richness $\left(\mathrm{SS}_{\mathrm{LR}}\right)$ & 1 & $Q$ or $M$ & .. & 0.00 \\
\hline Non-linear richness $\left(\mathrm{SS}_{\mathrm{NLR}}\right)$ & $S-2$ & $Q$ & 1.00 & 1.00 \\
\hline Partitioned species pools $\left(\mathrm{SS}_{Q}\right)$ & $Q-S$ & & 7.34 & 2.29 \\
\hline Species identity $\left(\mathrm{SS}_{I}\right)$ & $S-1$ & $M$ & 10.35 & 14.40 \\
\hline Species compositions $\left(\mathrm{SS}_{M}\right)$ & $M-Q-S-1$ & & $35.31 \leftrightarrow 24.96$ & 24.96 \\
\hline
\end{tabular}

Figure 2: The data were generated from equation (5) and are plotted in figure A1 in the online edition of the American Naturalist. Model A is $y_{j}=\beta_{0}+\beta_{Q} x_{Q}+\beta_{M} x_{M}$. In model B, species richness is entered before species identity (as in eq. [3]). In model C, species identity is entered before species richness (the order of the species identity term and the species richness terms is reversed). The arrows indicate how the error sums of squares $\mathrm{SS}_{Q}$ and $\mathrm{SS}_{M}$ are partitioned into sums of squares attributed to species richness and species identity, respectively. The dashed arrows indicate how the composition sum of squares is a composite of the effect of species identity and the effect of linear richness when species richness is entered into the model first. The degrees of freedom (df) associated with each source of variance and the appropriate denominator source of variance for $F$-tests (F-den.) are also indicated.

variance previously attributed to $\mathrm{SS}_{M}$ (model B in fig. 2), thus demonstrating that we have identified the correct error terms for $\mathrm{SS}_{R}$ and $\mathrm{SS}_{I}$, respectively. If the species identity term was added before the species richness term, then the value of $S_{I}$ increased and the value of $S_{R}$ decreased. When the richness term was divided into linear and nonlinear contrasts (as in eq. [3]), it is clear that the amount by which $\mathrm{SS}_{I}$ increased (and $\mathrm{SS}_{R}$ decreased) was exactly equal to $\mathrm{SS}_{\mathrm{LR}}$ (model $\mathrm{C}$ in fig. 2). As we noted above, the degree to which $\mathrm{SS}_{R}$ and $\mathrm{SS}_{I}$ overlap is therefore exactly $\mathrm{SS}_{\mathrm{LR}}$. Thus, if $\mathrm{SS}_{\mathrm{LR}}$ is entered into the model before $\mathrm{SS}_{I}$ and $\mathrm{SS}_{\mathrm{NLR}}$, $\mathrm{SS}_{I}$ and $\mathrm{SS}_{\mathrm{NLR}}$ are orthogonal, so their order of entry does not matter. See appendix B in the online edition of the American Naturalist for step-by-step instructions on how to conduct these analyses.

It is worth noting that a similar dependence exists between $\mathrm{SS}_{R}$ and $\mathrm{SS}_{I}$ on the one side and $\mathrm{SS}_{M}$ on the other side. As we have mentioned, a fully factorial design would include all possible compositions (i.e., all main effects and interactions of species identities). Even if we do not have all possible compositions, the rule still applies that as soon as differences between compositions are explained, all differences between richness levels and species identities are also explained. We therefore always list the compositions term after the richness and identity terms.

Finally, it should be noted that the variance due to nonlinear richness is derived from the partitioned species pools, whereas the variance due to species identity is derived from species compositions. Thus $\mathrm{SS}_{M}$ is the appro- priate denominator for $F$-tests involving $\mathrm{SS}_{R}$, whereas $\mathrm{SS}_{Q}$ is the denominator for $\mathrm{SS}_{I}$ (fig. 2).

\section{Interpretation of the Linear Model Coefficients}

In biodiversity manipulation experiments we are often interested in whether one species has a much greater effect on ecosystem functioning compared to the other species. We can easily obtain the impact of each species relative to the "average" species using our linear model framework by adding the linear effect of species richness before the individual species effects (i.e., in the order that they are entered in eq. [3]). As we have argued above, the first reason why we favor this formulation is that species identity $\left(\mathrm{SS}_{I}\right)$ and nonlinear species richness $\left(\mathrm{SS}_{\mathrm{NLR}}\right)$ are orthogonal once linear richness $\left(\mathrm{SS}_{\mathrm{LR}}\right)$ has been added to the model. The second reason is that $\sum_{i}^{S} \beta_{i}=0$. As a result, the $\beta_{i}$ coefficients become a measure of the effect of species $i$ on ecosystem functioning relative to the average species, where a positive coefficient indicates the species has an above-average contribution to function and a negative value denotes a below-average contribution. The third reason why we favor this formulation is that the nonlinear species richness coefficients give the degree to which levels of species richness deviate from the linear expectation. As such, we can use the sum of squares (or variance) of these coefficients as a surrogate for the importance of interactions among species. Significant nonlinear richness terms would be consistent with the hypothesis that species in- 
teractions are important in determining the shape of the biodiversity-ecosystem functioning relationship.

Other analyses are possible such as fitting the species coefficients before fitting linear richness. The key is that different hypotheses about species identity are being tested when linear richness is fitted before or after the other terms. If linear richness is added before the other terms, the hypothesis being tested is that the species effects are linear and equivalent and that any further effects of species richness represent deviations from linearity. Finally, it is worth noting that, although the species identity and nonlinear richness terms are orthogonal, the individual coefficients associated with each of $S$ species are not orthogonal.

\section{Ecological Scenarios}

The principle goal of the analysis that we have presented is to infer ecological mechanisms. To this end, we outline four ecological scenarios below and discuss how they can be interpreted using our approach. These are meant to be simple, contrived examples where it is possible to judge the performance of the analysis method in situations where we know how it should behave. Instructions on how to conduct these analyses are presented in appendix B.

\section{Scenario 1: Species Are Additive and Equivalent}

Consider first a situation where the species in a community have an equivalent effect on ecosystem functioning and are independent. This would be a community where none of the species compete for resources, share predators, or interact in any other way. If that is the case, the effect of every species on ecosystem functioning is additive. To avoid effects of different initial densities of species among richness levels, we use an additive design (i.e., there are initially twice as many individuals in two-species mixtures as in monocultures, four times as many in four-species mixtures as in monocultures, etc.). Note, however, that as long as the law of constant final yield applies (e.g., for plants), the following also applies to the substitutive design where the total initial number of individuals in a community is held constant (Schmid et al. 2002b). The consequence of noninteracting species is that ecosystem functioning is simply the sum of the ecosystem functioning of the monocultures of the species that constitute that composition. Here,

$$
\begin{aligned}
& y=\left(\sum_{i}^{s} b_{i} x_{i}\right), \\
& b_{i}=\frac{1}{6}
\end{aligned}
$$

where $y$ is the measured level of ecosystem functioning, $b_{i}$ is the actual effect of species $i$ on ecosystem functioning, and $x_{i}$ indicates whether species $i$ is present or absent in the composition. So, for example, for a composition containing species 1 , species 3 , and species 4 , the amount of ecosystem functioning would be calculated as $y=b_{1}+$ $b_{3}+b_{4}=3 / 6$. In a substitutive experiment, this would occur if the contribution of each species is density independent (i.e., each species at the end produced 1/6 at all richness levels irrespective of its starting density). When the results are plotted as a function of species richness, there is a linear relationship with no variation around the least squares line (fig. $3 A$ ). As we have discussed, there are several ways of modeling the results of scenario 1 . If we fit a regression model where the species effects are entered first and the species richness term is entered second, we obtain the regression equation

$$
y=0+\frac{1}{6} x_{1}+\frac{1}{6} x_{2}+\cdots+\frac{1}{6} x_{6}+0 x_{R} .
$$

From this equation, we would conclude that the species identity (the coefficients associated with each of the species) explained all of the variation in ecosystem functioning, while species richness $\left(x_{R}\right)$ explained none of the variation in ecosystem function (fig. 4A). In contrast, if species richness was entered before the species effects (as in eq. [3]), the linear richness term would explain all of the variation (see figs. $3 A, 4 A$ ), and the regression equation becomes

$$
y=0+\frac{1}{6} x_{\mathrm{LR}}+0 x_{1}+0 x_{2}+\cdots+0 x_{6}+0 x_{\mathrm{NLR}}
$$

The difference between equation (6) and equation (7) is that the species coefficients in equation (7) represent the effect of each species relative to the "average" species. For this formulation, the linear effect of species richness is not useful on its own; rather, we use the linear effect of species richness to give us species coefficients that are ecologically meaningful. Since the coefficient of every species is equal to 0 in equation (7), we can (correctly) conclude that the actual effect of every species on ecosystem functioning is equal to the average species, or in other words, every species has the same effect on functioning. In equation (7), the absolute effect of each species is $\varphi_{i}=\mu+\beta_{i}=$ $1 / 6+0$ (see eq. [5]), so the absolute effect of each species relative to the mean level of functioning is the same for both equation (6) and equation (7). Note that the effect of nonlinear richness is 0 (table 1 ). 


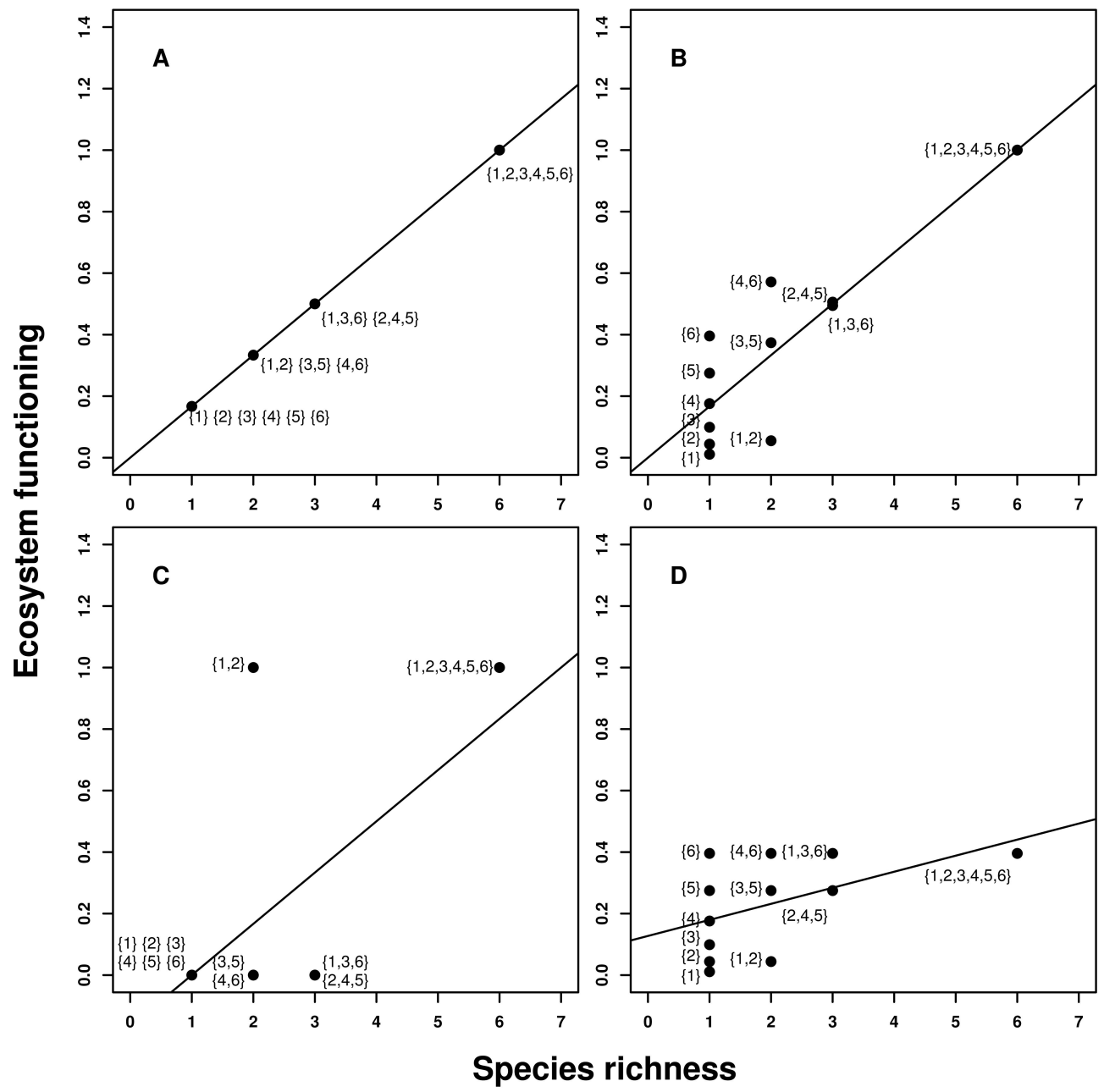

Figure 3: Relationship between species richness and ecosystem functioning for the four scenarios described in the text. $A$, Scenario 1: species are additive and equivalent. $B$, Scenario 2: species are additive and different. $C$, Scenario 3: species are obligate mutualists (facilitation). $D$, Scenario 4: species compete for a single resource (negative interactions). Each data point is one species composition, and the numbers show the identity of the species within each composition. For clarity, only one partitioned species pool is shown at each level of richness $(P=1$ in fig. 1$)$.

\section{Scenario 2: Species Are Additive and Different}

The analysis that we have outlined is also applicable if the species effects are not equivalent. For example, we can set the effect of each species on ecosystem functioning to be

$$
y=\left(\sum_{i}^{s} b_{i} x_{i}\right)
$$

where $b_{i}=i^{2} / 91$ and $i=1,2, \ldots, 6$.

The value of the denominator of $b_{i}$ is arbitrary but for convenience is taken to be 91 so that the summation $i^{2} / 91$ over all species is equal to unity. Unlike in equation (6), the species now have different effects on ecosystem functioning. For example, if species 1, 3, and 4 are present in composition $j$, the level of ecosystem functioning is $y=1^{2} / 91+3^{2} / 91+4^{2} / 91=26 / 91$. The results (fig. $3 B$ ) show that, as in scenario 1 , there is a linear relationship between species richness and ecosystem functioning but that there is now variation around the line of best fit (fig. $4 B$ ). When the species effects are added after the effect of linear richness, the least squares fit (see table 1) is

$$
\begin{aligned}
y= & 0+\frac{1}{6} x_{\mathrm{LR}}-0.156 x_{1}-0.123 x_{2}-0.068 x_{3} \\
& +0.009 x_{4}+0.108 x_{5}+0.229 x_{6}+0 x_{\mathrm{NLR}} .
\end{aligned}
$$

As in scenario 1, we can recover the actual species ef- 


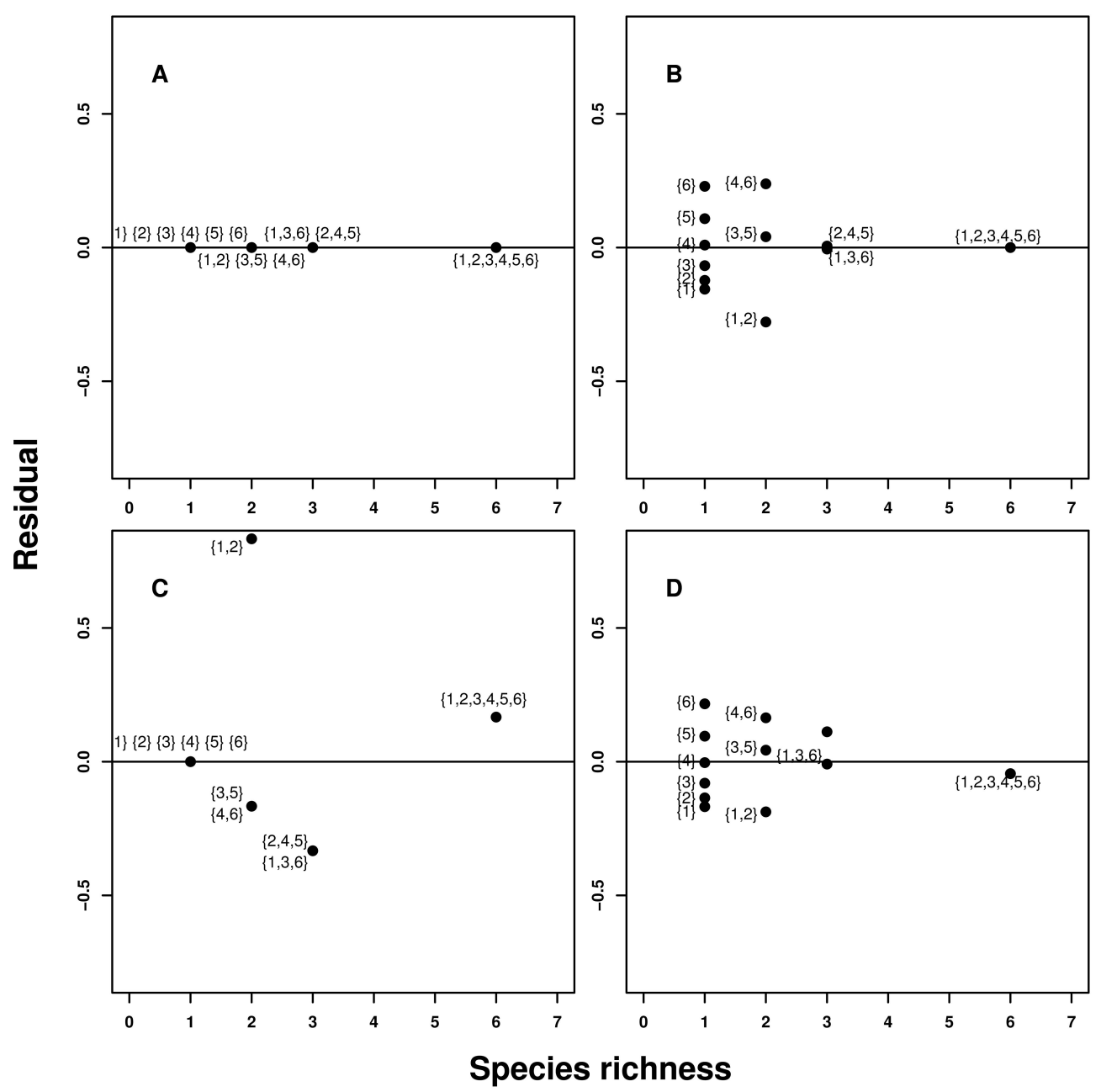

Figure 4: Relationship between species richness and the residuals of the model that includes only linear richness (i.e., residuals from the line fitted in fig. 2). Each data point is one species composition, and the numbers show the identity of the species within each composition. For clarity, only one partitioned species pool is shown at each level of richness $(P=1$ in fig. 1$)$.

fects. For example, the effect of species 5 is predicted to be (from eq. [9]) the sum of the effect of linear richness $(1 / 6)$ and of the coefficient associated with species 5 (0.108), which we find is equal to the "correct" effect of species 5 on ecosystem functioning: $1 / 6+0.108=5^{2} / 91$. As for scenario 1 , the $\beta_{i}$ coefficients give the average effect of every species on species richness because the coefficients sum to 0 . We would say, for example, that a composition that contains species 6 has a level of ecosystem functioning that is 0.229 higher than the situation where it was replaced with an "average" species. Similarly, we would expect a reduction in the level of ecosystem functioning of 0.229 if species 6 were replaced with an average species. This is clearly an important conclusion from the perspective both of the ecology of this community and of conservation or invasion biology, where estimating the average degree to which extinctions or invasions will influence functioning is of particular importance. As for scenario 1, the nonlinear effect of species richness $\left(\beta_{\mathrm{NLR}}\right)$ is 0 (table 1 ).

\section{Scenario 3: Two Species Interact}

We now consider a scenario where species interact. In the simplest situation, the level of ecosystem functioning is determined only by interactions among two species. For each composition, ecosystem functioning was calculated as

$$
y=b_{1 \times 2}\left(x_{1} x_{2}\right)
$$

where $b_{1 \times 2}$ is the coefficient for the interaction between 
Table 1: Results from the analysis of the four ecological scenarios

\begin{tabular}{lcccc}
\hline Linear model coefficient & Scenario 1 & Scenario 2 & Scenario 3 & Scenario 4 \\
\hline$\beta_{0}$ & 0 & 0 & -.167 & .123 \\
$\beta_{\mathrm{LR}}$ & .167 & .167 & .188 & .049 \\
$\beta_{I}=$ & & & & \\
$\beta_{1}$ & 0 & -.156 & .229 & -.107 \\
$\beta_{2}$ & 0 & -.123 & .249 & -.083 \\
$\beta_{3}$ & 0 & -.068 & -.079 & -.022 \\
$\beta_{4}$ & 0 & .009 & -.135 & -.021 \\
$\beta_{5}$ & 0 & .108 & -.135 & .053 \\
$\beta_{6}$ & 0 & .229 & -.130 & .180 \\
$\beta_{\mathrm{NLR}}=$ & & & & \\
$R=1$ & 0 & 0 & -.021 & -.005 \\
$R=2$ & 0 & 0 & .125 & .001 \\
$R=3$ & 0 & 0 & -.145 & .0224 \\
$R=6$ & 0 & 0 & .0417 & -.0188 \\
\hline
\end{tabular}

Note: The values of the linear model coefficients associated with the intercept $\left(\beta_{0}\right)$, linear richness $\left(\beta_{\mathrm{LR}}\right)$, the identity of the six species $\left(\beta_{I}\right)$, and nonlinear richness $\left(\beta_{\mathrm{NLR}}\right)$ are given for the four ecological scenarios described in the text. Scenario $1=$ species are additive and equivalent; scenario 2 = species are additive and different; scenario $3=$ species and are obligate mutualists (facilitation); scenario $4=$ species are different and compete for the same resource (negative interactions). Note that the species effects are nonzero only when species have different effects on functioning (scenarios 2, 3,4) and that nonlinear richness is nonzero when there are interactions among species (scenarios 3,4 ).

species 1 and species 2 . In the first instance we define $b_{1 \times 2}=1$, which describes a situation where ecosystem functioning is greater than 0 only when species 1 and 2 are present in a composition together. Ecologically, this might represent an obligate mutualism.

The results of this scenario are plotted in figures $3 C$ and $4 C$. There is a positive relationship between species richness and ecosystem functioning. Our analysis indicates (correctly) that the compositions that contain species 1 and 2 have an average greater level of functioning and that compositions that contain the other four species have an average lower level of functioning (table 1). For example, on average, the addition of species 1 to a composition will increase the level of ecosystem functioning by $-0.167+$ $0.188+0.229=0.250$. Since we know the underlying model that generated the data, we know that this increase in functioning actually reflects the proportion of compositions that contain species 2 . To get at the mechanisms involved, it is necessary to model the interactions among the species.

We have introduced the nonlinear richness term as a surrogate measure for species interactions. Once linear species richness is added to the model, nonlinear species richness is the effect of species richness independent of the species identity. We suggest that nonlinear species richness is a good proxy for estimating the importance of species interactions when there are insufficient degrees of freedom to actually estimate all of the interaction terms. This is hinted at in this particular example because, in contrast with the previous scenarios, the $\beta_{\mathrm{NLR}}$ coefficients are nonzero (table 1). It is straightforward to demonstrate how $\beta_{\mathrm{NLR}}$ and species interactions are related by varying the value of $b_{1 \times 2}$ in equation (10). The example shown in figure $3 C$ has $b_{1 \times 2}=1$. If $b_{1 \times 2}$ is varied between 0 and 1 , there is a linear relationship between $b_{1 \times 2}$ and $\beta_{\mathrm{NLR}}$ (see appendix B and fig. A2 in the online edition of the American Naturalist). Crucially, this relationship is not altered by altering the species main effects $\left(b_{1}, b_{2}, \ldots, b_{S}\right)$. Thus $\beta_{\mathrm{NLR}}$ appears to accurately reflect the importance of interactions among species independent of the species' main effects.

\section{Scenario 4: Species Are Additive and Interact}

We now consider a scenario where several species interact, in this instance by competing for resources. At one extreme, all six species compete for a single resource, which is a scenario that has frequently been used to illustrate the "selection effect" (Tilman 1999b). We set the "competitive ability" of each species to be as in equation (8) (i.e., the "competitive ability" of species $i$ is $i^{2} / 91$ ). If all of the species are competing for a single resource and it is assumed that each species starts at equal abundance and only a single species can persist on the resource, the species with the highest competitive ability will drive the others to extinction. Once the poorer competitors are driven to extinction, the level of functioning is set by the victor. We assumed that the level of ecosystem functioning associated 
with each species is proportional to their competitive ability. So for example, if a composition contains species 1, 2 , and 3 , it will be the case that species 3 drives the other two species to extinction and the level of ecosystem functioning will be $3^{2} / 91$. The scenario can be summarized by the following equation:

$$
y=\max \left\{\frac{1^{2}}{91} x_{1}, \frac{2^{2}}{91} x_{2}, \ldots, \frac{6^{2}}{91} x_{6}\right\}
$$

where $\max \{\bullet\}$ is the maximum competitive ability that is present in each composition. As for the other scenarios, the compositions shown in figure 1 are used. As for the previous examples, we first fit linear species richness (fig. $3 D$ ) and then estimate the impact of each species relative to the average species by fitting the individual species effects (table 1). As for scenario 3, the nonlinear richness coefficients are nonzero (table 1 ).

There is again a positive relationship between diversity and functioning. In the language of the biodiversityecosystem functioning literature, there is no effect of biodiversity per se, because the level of functioning is set by which species are present in a composition rather than by the number of species that are present. In the language of our analysis, we would say that only interactions among species are important. If we could describe this situation fully, we would likely find that all of the two-, three-, and $S$-way interactions are significant. We cannot estimate all of the interaction terms but instead use $\beta_{\mathrm{NLR}}$ as a surrogate.

We predicted that $\beta_{\mathrm{NLR}}$ would approach 0 as interactions among species become less important. We have performed a simple simulation where the number of interacting species decreased from all six (as above) to 0 (as in scenario 2). If species' niches either overlap completely or do not overlap at all, we can vary the number of overlapping niches $(k)$ from 0 to 6 . Ecosystem functioning is then calculated as

$$
y=\max \left\{b_{1} x_{1}, \ldots, b_{k} x_{k}\right\}+\sum_{j=k+1}^{6} b_{j} x_{j}
$$

where the coefficients ( $b s$ ) are defined as in equation (8). In this list of scenarios, the species are completely complementary when $k=0$, and their niches completely overlap when $k=6$. As predicted, this is mirrored by an increase in $\beta_{\mathrm{NLR}}$ (fig. 5). This scenario also emphasizes another advantage of this experiment design: since every species is included at each richness level, the functioning of the best community at each richness level is constant. This result is not guaranteed in other designs and would be important especially if one cannot conduct the additive partitioning analyses of Loreau and Hector (2001).

\section{Application}

We applied the method to two data sets. The first is from a published study that manipulated 72 bacterial strains and measured community respiration (Bell et al. 2005). The second is from an unpublished experiment that manipulated six plant species and measured community biomass (see app. C in the online edition of the American Naturalist). As previously documented, bacterial community respiration increased with increasing bacterial diversity (fig. 6A). Although a few of the individual species effects were significant (fig. 6B) and species identity was in general significant, much more of the variation was explained by nonlinear richness (fig. 6C). In contrast, there was little apparent relationship between diversity and functioning for the plant experiment (fig. 6D). Relative to the number of species that were manipulated, many of the species were significantly associated with relatively high (Lolium perenne, Lolium arundinaceum) or low (Agrostis stolonifera, Poa pratensis) levels of functioning (fig. 6E). Overall, there was a highly significant effect of species identity but no significant effect of nonlinear richness (fig. $6 F$ ), confirming that the level of functioning was dependent largely on the identity of the species present in the community.

The method we have described therefore highlights the disparities between these communities. The bacterial community consists of a set of interacting species where no single species is able to dominate. In the plant community, interactions among the constituent species are relative unimportant. Rather, the level of functioning is determined principally by the identity of the species that constitute each mixture rather than the species richness of the mixture.

\section{Discussion}

The approach that we describe above is intended to provide an alternative for designing and analyzing biodiversity-ecosystem functioning experiments. The intention is not to supplant current methods but rather to develop methods around the data that can be collected rather than vice versa. Current analyses require knowing the relative contribution of every species to functioning (Loreau and Hector 2001; Fox 2006) and the functioning of each species in monoculture (Loreau and Hector 2001).The current approach makes it possible to estimate the importance of interactions among species when these data are unavailable. So long as the experiment is designed as we have described, all that is required is the initial composition of the communities and an estimate of total ecosystem functioning. Designing the experiment according to the random partition design endows the analysis with certain desirable properties, including orthogonality of the summed species identity and nonlinear richness terms. 


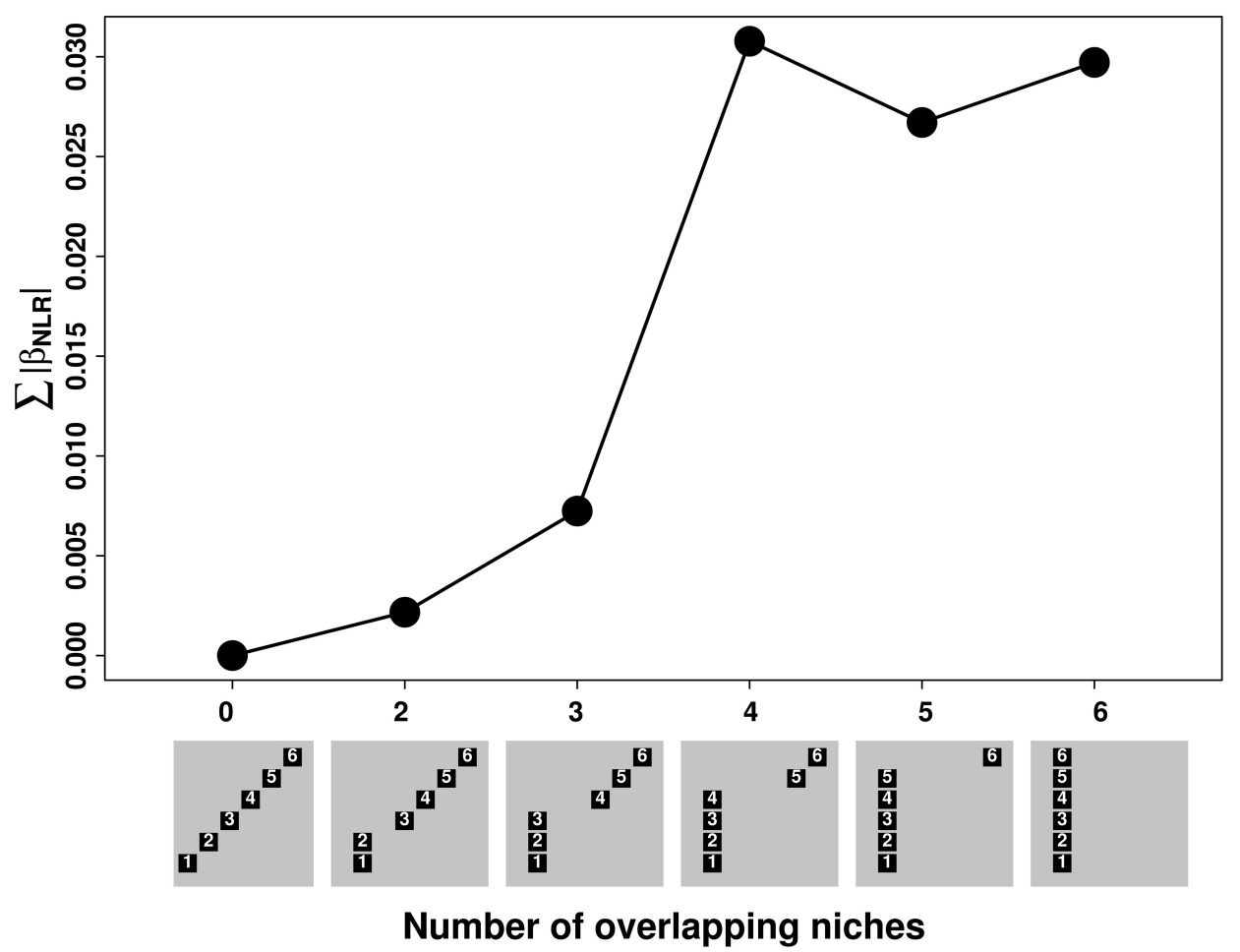

Figure 5: Relationship between the number of species interactions (i.e., the number of overlapping niches) and the nonlinear richness term ( $\left.\beta_{\mathrm{NLR}}\right)$. The numbered panels below the $X$-axis illustrate which species have overlapping or nonoverlapping niches, where the numbers denote the identity of the species. Overlap along the horizontal axis indicates that those species have overlapping niches. There is no niche overlap in the leftmost panel, whereas all species have overlapping niches in the rightmost panel.

We believe the method presented here makes the good use of data particularly in situations where the functional contributions of species are unknown. We would argue that research efforts have been devoted almost exclusively (Balvanera et al. 2006) on plant standing biomass to the detriment of studying considerably more important functions simply because it is easy to measure and to break down into functional contributions. At the very least, the method outlined here allows interpretation of data in the absence of knowledge of functional contributions.

There is, in addition, a separate class of ecosystem functions that constitute emergent properties since they can be measured only for the community as a whole rather than for the constituent species (Fox and Harpole 2008). These include, for example, the stability (Wardle and Grime 2003; Ptacnik et al. 2008) or the resistance to invasion (Arenas et al. 2006) of a community. By definition, these cannot be broken down into functional contributions, so it has been suggested that they should be viewed in a fundamentally different manner (Fox and Harpole 2008). No such separation is required so far as the current analysis is concerned. Although it would typically make little sense to attribute a portion of, for example, invasion resistance to a specific species, in our analysis the marginal species identity effects indicate whether a particular species is associated with invasion resistance to a greater or lesser extent than the average species. The question of why certain species are associated with high/low levels of functioning is more difficult to ascertain and would require further experimentation. Although these are purely statistical properties, it is clearly of interest to calculate these properties to understand how they are affected (on average) by the exclusion or inclusion of particular species.

One of the key controversies in biodiversity-ecosystem functioning research is that mechanisms must be inferred at the analysis stage. Two "effects," the selection effect and the complementarity effect, have drawn particular attention (Tilman 1999b). One of the benefits of the current design is that every species is given an equal chance to contribute to the selection and complementarity effect, whereas this would not be the case for other designs. In addition, nonoverlapping species compositions avoid a purely statistical variance-reduction effect.

Scenarios 1 and 2 demonstrate that our linear model approach is well suited for describing situations where species are completely complementary. Not only is the analysis 

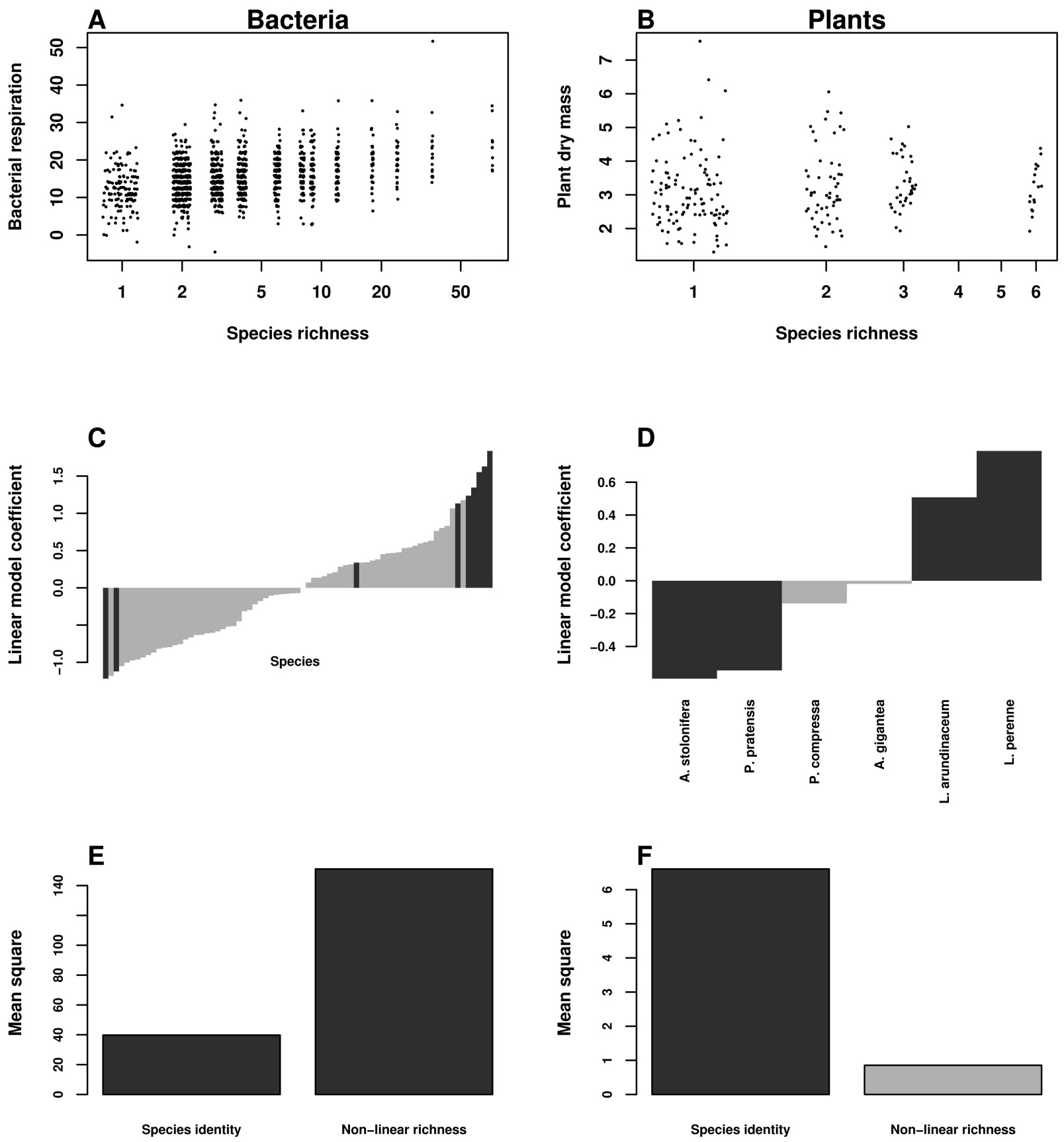

Figure 6: Application of the linear model method to experiments with bacteria. Relationship between species richness and ecosystem functioning of bacterial respiration $\left(\mu \mathrm{g} \mathrm{CO} \mathrm{mL}^{-1} ; A\right)$ and plant biomass (mg dry mass; $B$ ). Data points have been displaced slightly along each axis for clarity. The middle row indicates the linear model coefficients associated with each of the 72 bacterial species $(C)$ or six plant species $(D)$. The bottom row indicates the contribution of species identity and nonlinear species richness for bacteria $(E)$ and plants $(F)$. The methodology of each of the experiments is in appendix $\mathrm{C}$ in the online edition of the American Naturalist. 
able to identify that all species are complementary $\left(\beta_{\mathrm{NLR}}=0\right)$, the analysis also identifies the relative impact of each species on functioning and so provides crucial information about expected levels of functioning should one of the species be added or lost from a composition. Scenario 3 gives an example of positive interactions, whereas scenario 4 is an example of negative interactions. It is at this point that the comparison with methods derived from the Price equation (Loreau and Hector 2001) breaks down. Positive interactions, such as facilitation (scenario 3), would be considered part of complementarity effects in the additive partitioning method, whereas here we consider it the positive interaction of two species identity effects.

The current design does not explicitly examine the relative importance of selection effects. However, if only a subset of the pool of available species interacts, there will be a selection effect because the interacting species are more likely to occur together at high levels of species richness. For example, if two species compete for resources, the best competitor will persist, and so the level of ecosystem functioning will be set by the victor. The likelihood that the best competitor will be included in a composition increases with increasing species richness, so the level of ecosystem functioning increases with increasing species richness. In the same way, any ecological interaction (competition, predation, facilitation, etc.) will result in a relationship between species richness and ecosystem functioning due to the increased probability that the interacting species will be in a composition together at higher levels of diversity. The $\beta_{\mathrm{NLR}}$ coefficient provides an estimate of the importance of species interactions and so can directly measure the importance of nonlinearity of species identity effects. When $\beta_{\mathrm{NLR}}$ is high, the selection effect as defined here (i.e., which includes facilitation) will be important in determining the level of functioning because species interactions are important in determining functioning, whereas when $\beta_{\mathrm{NLR}}$ is near 0 , complementarity (excluding facilitation) will determine functioning so long as the individual species coefficients are significant. Contrasting the relative importance of species interactions versus their additive effects might prove a profitable avenue for future biodiversity-ecosystem functioning studies.

Rather than manipulating species richness, a number of studies have manipulated functional group richness and measured ecosystem functioning (Symstad et al. 2000; Wardle and Zackrisson 2005; Hoehn et al. 2008; Griffin et al. 2009). The current approach can also be applied to experiments using functional groups such that every functional group is selected without replacement at each level of functional group richness. If the random partition design is applied in this way to functional group richness, the linear model approach we have described will bear the same properties for the functional groups as it does for manipulations of species. For example, in an experiment designed in this manner, the functional group identity effects would be orthogonal to nonlinear functional group richness. However, it is unclear to us whether the approach can be used to simultaneously assess the roles of functional group richness and species richness; this might prove to be a fruitful avenue for future research.

Finally, it should be stressed that in our method, ecological mechanisms are implied rather than deduced. It will always be the case that to conclusively demonstrate interactions among species (or lack thereof) will require knowledge of functional contributions of each species. This should not prevent us from studying situations where this information is unavailable. The analysis and design described in this article provide a new starting point for interpreting biodiversity-ecosystem function experiments.

\section{Acknowledgments}

We are indebted to B. Silverman, who provided substantial input during the initial stages of this investigation, and to J. Fox and several anonymous reviewers who provided comments on an earlier version of the manuscript.

\section{Literature Cited}

Aarssen, L. W. 1997. High productivity in grassland ecosystems: effected by species diversity or productive species? Oikos 80:183-184.

Arenas, F., I. Sanchez, S. J. Hawkins, and S. R. Jenkins. 2006. The invasibility of marine algal assemblages: role of functional diversity and identity. Ecology 87:2851-2861.

Balvanera, P., A. Pfisterer, N. Buchmann, J.-S. He, D. Raffaelli, and B. Schmid. 2006. Quantifying the evidence for biodiversity effects on ecosystem functioning and services. Ecology Letters 9:1146-1156.

Bell, T., J. A. Newman, B. S. Silverman, S. L. Turner, and A. K. Lilley. 2005. The contribution of species richness and composition to bacterial services. Nature 436:1157-1160.

Cardinale, B. J., D. S. Srivastava, J. E. Duffy, J. P. Wright, A. L. Downing, M. Sankaran, and C. Jouseau. 2006. Effects of biodiversity on the functioning of trophic groups and ecosystems. Nature 443:989-992.

Fox, J. W. 2005. Interpreting the "selection effect" of biodiversity on ecosystem function. Ecology Letters 8:846-856.

. 2006. Using the Price equation to partition the effects of biodiversity loss on ecosystem function. Ecology 87:2687-2696.

Fox, J. W., and W. S. Harpole. 2008. Revealing how species loss affects ecosystem function: the trait-based Price equation partition. Ecology 89:269.

Griffin, J. N., V. Mendez, A. F. Johnson, S. R. Jenkins, and A. Foggo. 2009. Functional diversity predicts overyielding effect of species combination on primary productivity. Oikos 118:37-44.

Harper, J. L. 1977. Population biology of plants. Academic Press, London.

Hector, A., B. Schmid, C. Beierkuhnlein, M. C. Caldeira, M. Diemer, P. G. Dimitrakopoulos, J. A. Finn, et al. 1999. Plant diversity and productivity experiments in European grasslands. Science 286: 1123-1127.

Hector, A., J. Joshi, S. P. Lawler, E. M. Spehn, and A. Wilby. 2001. 
Conservation implications of the link between biodiversity and ecosystem functioning. Oecologia (Berlin) 129:624-628.

Hodgson, D. J., P. B. Rainey, and A. Buckling. 2002. Mechanisms linking diversity, productivity and invasibility in experimental bacterial communities. Proceedings of the Royal Society B: Biological Sciences 269:2277-2283.

Hoehn, P., T. Tscharntke, J. M. Tylianakis, and I. Steffan-Dewenter. 2008. Functional group diversity of bee pollinators increases crop yield. Proceedings of the Royal Society B: Biological Sciences 275: 2283-2291.

Hooper, D. U., F. S. Chapin III, J. J. Ewel, A. Hector, P. Inchausti, S. Lavorel, J. H. Lawton, et al. 2005. Effects of biodiversity on ecosystem functioning: a concensus of current knowledge. Ecological Monographs 75:3-35.

Huston, M. A. 1997. Hidden treatments in ecological experiments: re-evaluating the ecosystem function of biodiversity. Oecologia (Berlin) 110:449-460.

Huston, M. A., and A. C. McBride. 2002. Evaluating the relative strengths of biotic versus abiotic controls on ecosystem processes. Pages 47-60 in M. Loreau, S. Naeem, and P. Inchausti, eds. Biodiversity and ecosystem functioning: synthesis and perspectives. Oxford University Press, Oxford.

Huston, M. A., L. W. Aarssen, M. P. Austin, B. S. Cade, J. D. Fridley, E. Garnier, J. P. Grime, et al. 2000. No consistent effect of plant diversity on productivity. Science 289:1255.

Loreau, M., and A. Hector. 2001. Partitioning selection and complementarity in biodiversity experiments. Nature 412:72-76.

Petchey, O. L. 2003. Integrating methods that investigate how complementarity influences ecosystem functioning. Oikos 101:323-330.

Ptacnik, R., A. G. Solimini, T. Andersen, T. Tamminen, P. Brettum, L. Lepisto, E. Willen, et al. 2008. Diversity predicts stability and resource use efficiency in natural phytoplankton communities. Proceedings of the National Academy of Sciences of the USA 105: 5134-5138.

Roscher, C., J. Schumacher, J. Baade, W. Wilcke, G. Gleixner, W. W. Weisser, B. Schmid, et al. 2004. The role of biodiversity for element cycling and trophic interactions: an experimental approach in a grassland community. Basic and Applied Ecology 5:107-121.

Roscher, C., V. M. Temperton, M. Scherer-Lorenzen, M. Schmitz, J. Schumacher, B. Schmid, N. Buchmann, et al. 2005. Overyielding in experimental grassland communities-irrespective of species pool or spatial scale. Ecology Letters 8:419-429.
Salles, J. F., F. Poly, and X. Le Roux. Forthcoming. Community niche predicts the functioning of denitrifying bacterial assemblages. Ecology.

Schmid, B., A. Hector, M. A. Huston, P. Inchausti, I. Nijs, P. W. Leadley, and D. Tilman. 2002a. The design and analysis of biodiversity experiments. Pages 61-75 in M. Loreau, S. Naeem, and P. Inchausti, eds. Biodiversity and ecosystem functioning: synthesis and perspectives. Oxford University Press, Oxford.

Schmid, B., J. Joshi, and F. Schlapfer. 2002b. Empirical evidence for biodiversity-ecosystem functioning relationships. Pages 120-150 in A. P. Kinzig, S. W. Pacala, and D. Tilman, eds. Functional consequences of biodiversity: empirical progress and theoretical extensions. Princeton University Press, Princeton, NJ.

Srivastava, D. S., and M. Vellend. 2005. Biodiversity-ecosystem function research: is it relevant to conservation? Annual Review of Ecology, Evolution, and Systematics 36:267-294.

Symstad, A. J., E. Siemann, and J. Haarstad. 2000. An experimental test of the effect of plant functional group diversity on arthropod diversity. Oikos 89:243-253.

Tilman, D. 1999a. Diversity and production in European grasslands. Science 286:1099-1100.

- 1999b. The ecological consequences of changes in biodiversity: a search for general principles. Ecology 80:1455-1474.

Tilman, D., S. Naeem, J. Knops, P. Reich, E. Siemann, D. Wedin, M. Ritchie, et al. 1997. Biodiversity and ecosystem properties. Science 278:1866-1867.

Wacker, L., O. Baudois, E. Eichenberger-Glinz, and B. Schmid. 2008. Environmental heterogeneity increases complementarity in experimental grassland communities. Basic and Applied Ecology 9:467474 .

Wardle, D. A. 1998. A more reliable design for biodiversity study? Nature 394:30.

. 1999. Is "sampling effect" a problem for experiments investigating biodiversity-ecosystem function relationships? Oikos 87:403-407.

Wardle, D. A., and J. P. Grime. 2003. Biodiversity and stability of grassland ecosystem functioning. Oikos 100:622-623.

Wardle, D. A., and O. Zackrisson. 2005. Effects of species and functional group loss on island ecosystem properties. Nature 435:806-810.

Associate Editor: Peter J. Morin Editor: Donald L. DeAngelis 